\title{
BMJ Open Injection therapy for base of thumb osteoarthritis: a systematic review and meta-analysis
}

\author{
Nicholas Riley, ${ }^{1}$ Martinique Vella-Baldacchino, ${ }^{1}$ Neal Thurley, ${ }^{2}$ Sally Hopewell, ${ }^{3}$ \\ Andrew J Carr, ${ }^{4}$ Benjamin John Floyd Dean ${ }^{4}$
}

To cite: Riley N, VellaBaldacchino M, Thurley N, et al. Injection therapy for base of thumb osteoarthritis: a systematic review and meta-analysis. BMJ Open 2019;9:e027507. doi:10.1136/ bmjopen-2018-027507

- Prepublication history and additional material for this paper are available online. To view these files, please visit the journal online (http://dx.doi. org/10.1136/bmjopen-2018027507).

Received 26 October 2018 Revised 08 August 2019 Accepted 09 August 2019

Check for updates

(c) Author(s) (or their employer(s)) 2019. Re-use permitted under CC BY-NC. No commercial re-use. See rights and permissions. Published by BMJ.

${ }^{1}$ Oxford University Hospitals NHS Foundation Trust Nuffield Orthopaedic Centre, Oxford, UK ${ }^{2}$ University of Oxford Health Care Libraries, Oxford, UK

${ }^{3}$ Oxford Clinical Trials Research Unit, Centre for Statistics in

Medicine, Oxford, UK

${ }^{4}$ NDORMS, University of Oxford, Oxford, UK

Correspondence to

Dr Benjamin John Floyd Dean; bendean1979@gmail.com

\section{ABSTRACT}

Objective To evaluate the effectiveness of injection-based therapy in base of thumb osteoarthritis.

Design Systematic review and meta-analysis.

Data sources MEDLINE and EMBASE via OVID, CINAHL and SPORTDiscus via EBSCO were searched from inception to 22 May 2018.

Study selection Randomised controlled trials (RCTs) and non-RCTs of adults with base of thumb osteoarthritis investigating an injection-based intervention with any comparator/s.

Data extraction and analysis Data were extracted and checked for accuracy and completeness by pairs of reviewers. Primary outcomes were pain and function. Comparative treatment effects were analysed by random-effects model for short-term and medium-term follow-up.

Results In total, 9 RCTs involving 504 patients were identified for inclusion. All compared different injectionbased therapies with each other, no studies compared an injection-based therapy with a non-injection-based intervention. Twenty injection-based intervention groups were present within these nine trials, consisting of hyaluronic acid $(n=9)$, corticosteroid $(n=7)$, saline placebo $(n=3)$ and dextrose $(n=1)$. Limited meta-analysis was possible due to the heterogeneity in the injections and outcomes used, as well as incomplete outcome data. Meta-analysis of two RCTs (92 patients) demonstrated reduced Visual Analogue Scale pain on activity with corticosteroid versus hyaluronic acid (mean difference (MD) $-1.32,95 \% \mathrm{Cl}-2.23$ to -0.41 ) in the medium term, but no differences in other measures of pain or function in the short term and medium term. Overall, the available evidence does not suggest that any of the commonly used injection therapies are superior to placebo, one another or a non-injection-based comparator.

Conclusion Current evidence is equivocal regarding the use of injection therapy in base of thumb osteoarthritis, both in terms of which injection-based therapy is the most effective and in terms of whether any injection-based therapy is more effective than other non-injection-based interventions. Given limited understanding of both the short-term and long-term effects, there is a need for a large, methodologically robust RCT investigating the commonly used injection therapies and comparing them with other therapeutic options and placebo.

PROSPERO registration number CRD42018095384.

\section{Strengths and limitations of this study}

- This systematic review was conducted in accordance with Preferred Reporting Items for Systematic Reviews and Meta-Analyses guidelines.

- Using a comprehensive search strategy, all available and relevant published literature was included for evaluation.

- There are several fairly consistent methodological flaws present within the trials included in this review: the studies are all small single-centre studies which appear significantly underpowered, there is a consistent failure to clearly prespecify and state a primary outcome measure and the use of concomitant treatments has not been pragmatic.

- The meta-analysis has been limited by the lack of studies providing adequate outcome data.

\section{INTRODUCTION}

Base of thumb osteoarthritis is a common condition that is frequently associated with significant levels of pain, dysfunction and disability. ${ }^{12}$ The key risk factors include increasing age and female gender. ${ }^{2}$ The majority of base of thumb pain is managed in primary care or at primary care interface musculoskeletal services by physiotherapists, occupational therapists and general practitioners. The aim of treatment is to improve pain and function, and usual care often encompasses the current guidance from the British Society of Surgery for the Hand advising avoidance of painful activities, analgesia, splintage and steroid injections, with surgery considered to be a 'last resort'. Usual care is likely to be highly variable, while there is some evidence which suggests that a majority of patients respond to non-surgical interventions and avoid surgery. ${ }^{4}$

There is a lack of high-quality evidence to guide the non-surgical management of base of thumb osteoarthritis, ${ }^{56}$ and the existing literature demonstrates no clear answer as regard the effectiveness of injection-based interventions such as corticosteroid. ${ }^{7}$ Steroid 
injections have been more widely studied in treating shoulder pain in which a short-term benefit over placebo has been demonstrated, ${ }^{8}$ however concerns remain over their long-term clinical effects. ${ }^{9} 10$

Given this lack of clarity, our aim was to perform a systematic review of the effectiveness of injection-based interventions compared with any comparator/s for base of thumb osteoarthritis in terms of patient-reported outcome measures and to assess the rates of adverse outcomes associated with these interventions.

\section{METHODS}

The systematic review is reported in accordance with the Preferred Reporting Items for Systematic Reviews and Meta-Analyses statement, using methodology described in the Cochrane Handbook for Systematic Reviews of Interventions. The protocol was developed prospectively and peer reviewed locally before registration on the PROSPERO database (CRD42018095384).

\section{Data sources and searches}

A comprehensive search strategy was created in collaboration with a research librarian (NT) and was designed to capture all relevant articles pertaining to injection-based inventions for base of thumb osteoarthritis (see online supplementary material 1). The full search strategy is detailed on the PROSPERO website. The search strategy was applied to the following bibliographic databases from database inception until 22 May 2018: MEDLINE and EMBASE via OVID, CINAHL and SPORTDiscus via EBSCO from database inception until 22 May 2018.

\section{Inclusion/exclusion criteria}

The inclusion and exclusion criteria were defined prospectively during the protocol stage. Any prospective study relating to an injection-based intervention for base of thumb osteoarthrtitis (trapeziometacarpal) was included. Studies had to contain an injection-based intervention and a comparator/s (ie, both non-randomised controlled trials (non-RCT), and RCTs, including semi-randomised/quasi-randomised, cluster randomised trials and comparative case series). Studies were excluded if patients were under the age of 18 years and if treatment was for inflammatory arthritis such as rheumatoid. Review articles, studies not published as a full article (conference abstracts) and case studies were excluded.

\section{Selection of studies}

Duplicates were removed and relevant studies identified from the search were imported into Covidence for screening. Studies were independently screened by title and abstract by two authors (BJFD and MV-B). The references of all included studies and all relevant review articles on the topic were also reviewed to identify other potential studies for inclusion. This was followed by a fulltext evaluation of the selected studies from the first selection step by these authors. Disagreement between the two reviewers was solved by consensus involving a third author (NR).

\section{Data extraction}

Two reviewers (MV-B and BJFD) independently extracted data. Data were extracted using a custom data extraction sheet in Covidence (http://www.covidence.org). The custom data extraction sheet was specifically designed to extract data relating to study design, details relating to the interventions undertaken and details regarding the other treatment undergone by trial participants alongside the described interventions. Any inconsistencies between the two reviewers' forms were resolved by consensus discussion. A third review (NR) was available for any disagreement that could not be resolved by this initial discussion.

If data were not available from full-text articles or trial registrations, the authors were contacted to provide this information. If the authors were not contactable as regard additional data, then this aspect of the study was excluded from the data synthesis. If contactable authors did not respond to initial requests, they were sent two subsequent reminders over a minimum of 6 weeks. If there was still no response for the additional data, then this aspect of the study was excluded from the data synthesis.

\section{Risk of bias assessment}

Included studies were assessed for risk of bias by two independent raters (BJFD and MV-B) using the Cochrane Collaboration's tool for assessing risk of bias in randomised trials. ${ }^{11}$ This followed the description in the Cochrane Handbook for Systematic Review of Interventions, V.5.1 (Part 2: 8.5.1). ${ }^{11}$ Any disagreements between ratings were resolved by discussion between the raters. A third party (NR) was available in any case where disagreements persisted after discussion.

\section{Outcomes}

Patient-reported pain and function were the primary outcomes of interest, adverse events were also recorded. A priori we defined end points as short term (1 week up to but not including 3 months), medium term (3 months up to and including 6 months) and long term (above 6 months). Where outcome data were available for more than one time point in each time category (short, medium and long term) then the data for the longest time point was used.

\section{Data analysis}

Descriptive analysis was performed for all demographic, intervention and outcome data to facilitate narrative interpretation and comparison across studies. Details regarding concomitant treatments in the different study arms such as the use of analgesics, splintage and physiotherapy were also recorded. Due to limited data, a direct-comparison meta-analysis was only performed for corticosteroid versus hyaluronic acid for pain (ie, Visual Analogue Scale (VAS) rest and VAS activity) and function (ie, grip strength and tip pinch strength). This was the only area in which data were available for similar time 


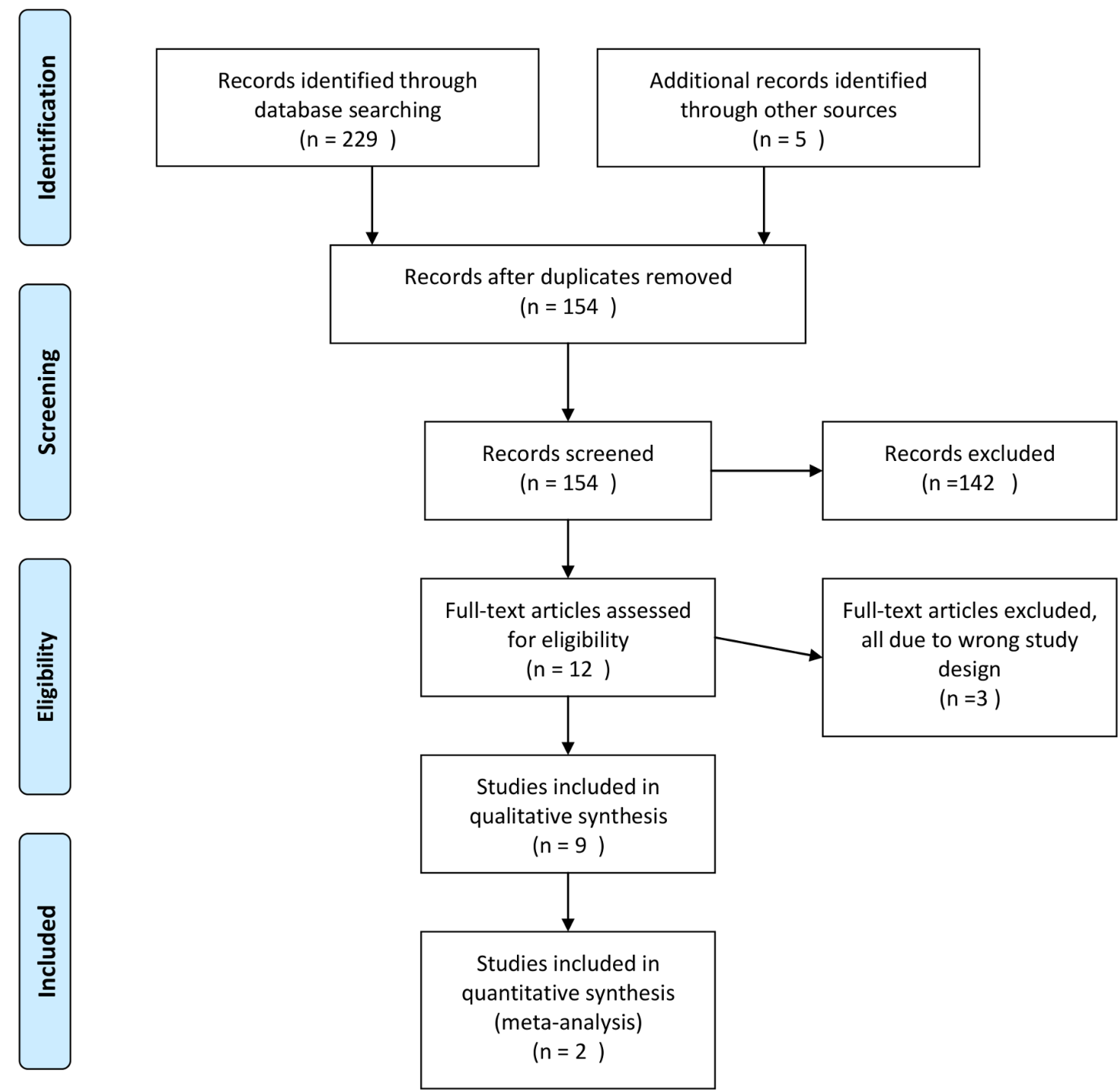

Figure 1 Preferred Reporting Items for Systematic Reviews and Meta-Analyses flow diagram. (Source) Reproduced from Moher et al, $2009^{29}$

points, outcomes and interventions across two or more studies. Mean difference was used for the meta-analysis of VAS pain and standardised mean difference was used for the meta-analysis of function (grip strength and tip pinch strength). Statistical heterogeneity was determined according to Cochrane interpretation ( $\mathrm{I}^{2}>75 \%$ considerable heterogeneity). Analysis was performed using RevMan using both random-effects and fixed-effects models.

\section{Patient and public involvement}

Patients have not been involved in this review.

\section{RESULTS}

\section{Study selection}

A total of 229 studies were identified by the search, after duplicates were removed. After screening by full-text, nine RCTs were identified as eligible for inclusion (figure 1). The number of studies identified and excluded at each stage is detailed in figure 1.

\section{Study characteristics}

Study characteristics of the included trials including the interventions and comparators are provided in table 1. Seven RCTs contained two injection therapy treatment groups, while two contained three injection therapy treatment groups. The most common comparison was steroid versus hyaluronic acid (four RCTs). ${ }^{12-15}$ Other trials compared placebo with hyaluronic acid, ${ }^{16}$ steroid versus hyaluronic acid versus placebo, ${ }^{17}$ steroid versus dextrose ${ }^{18}$ steroid versus placebo ${ }^{19}$ and three different hyaluronic acid injection regimes. ${ }^{20}$ There was wide variation in terms of the number of injections, drugs and doses used, as well the mode of injection delivery (anatomical as opposed to guided by ultrasound or fluoroscopically). Only three RCTs performed injections under guidance, two of these used fluoroscopic guidance ${ }^{1920}$ and one ultrasound. ${ }^{14}$ No RCTs compared injection with a non-injection compactor.

Table 2 details the inclusion and exclusion criteria, the basic demographics of the intervention and comparator 


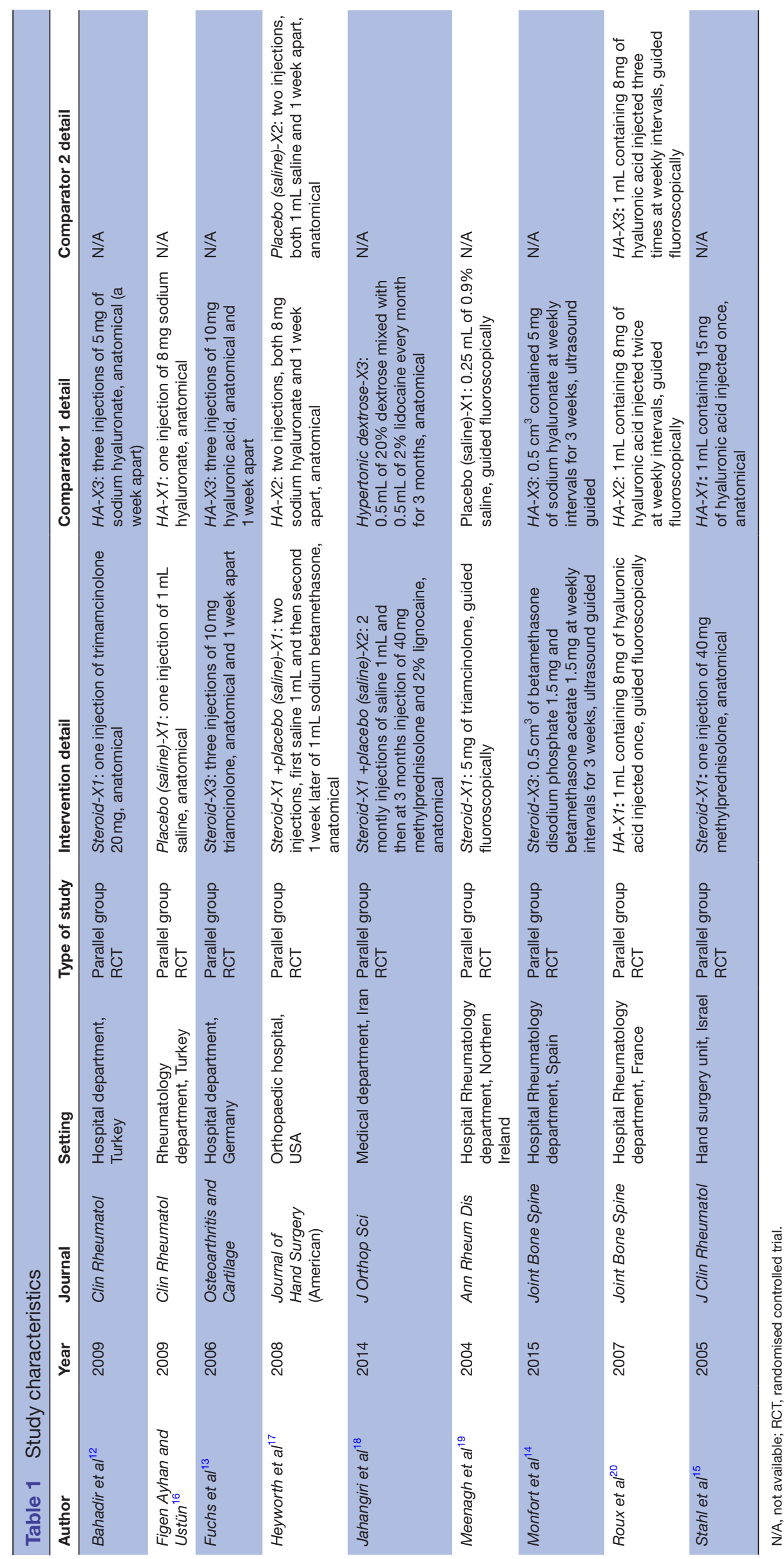




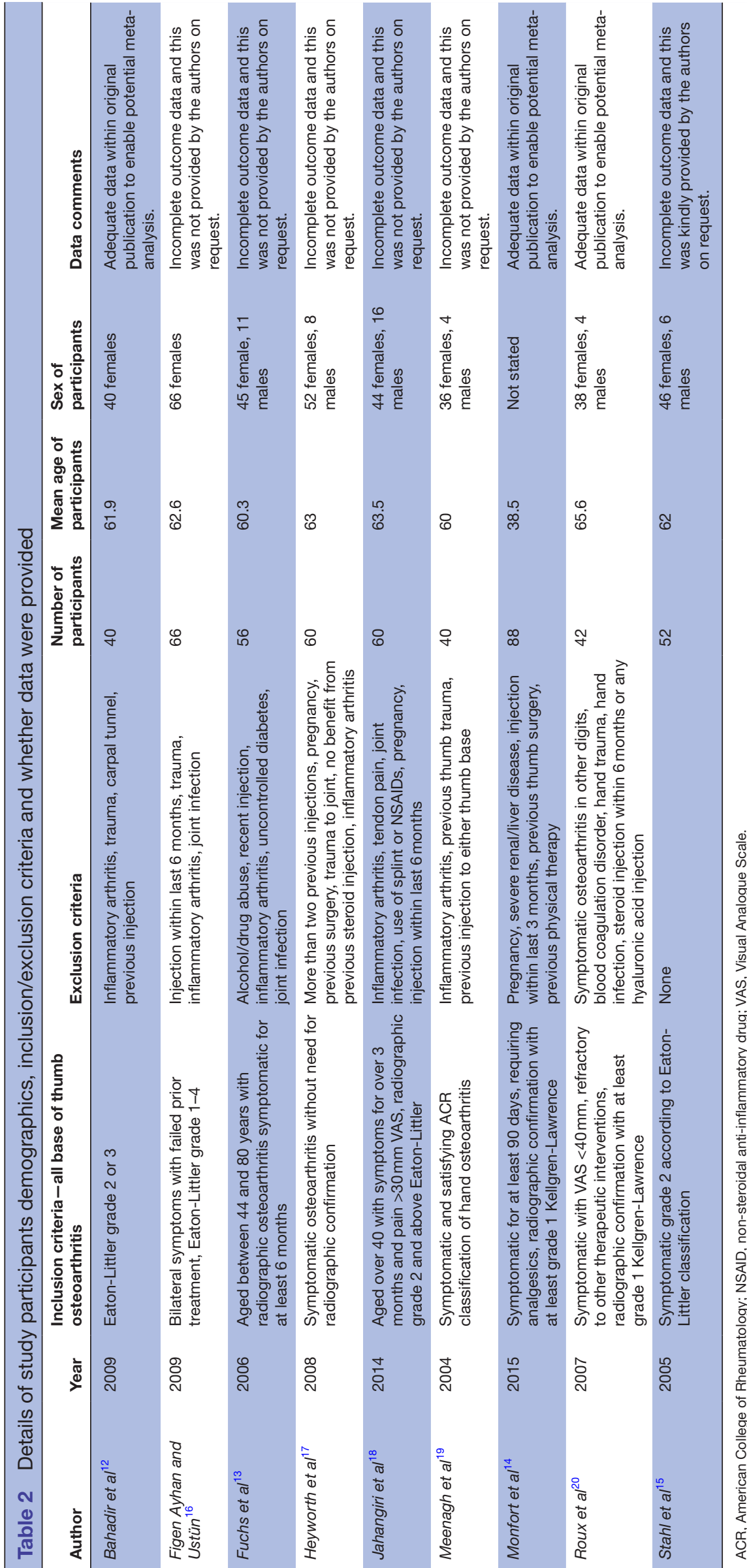


groups as well as details relating to the outcome data. Inclusion and exclusion criteria were highly variable. All trials were solely related to adults with symptomatic base of thumb osteoarthritis, most specified a particular radiographic grading as an inclusion criterion, either the classification by Eaton and Littler or Kellgren and Lawrence were used. Two trials did not specify a particular grade of base of thumb osteoarthritis. ${ }^{17}{ }^{19}$ One trial specified the need for bilateral symptoms as one side received steroid and the other placebo injection. ${ }^{16}$ Two trials were exclusively of females. ${ }^{12} 16$ The remaining trials included a minority of men, while one did not state the gender breakdown. ${ }^{14}$ The mean age of participants was close to 60 years, other than the study by Monfort $e t$ al, ${ }^{14}$ which had a mean age of 38.5 years. Only three trials contained adequate data within the published text for undertaking further analysis. ${ }^{12}{ }^{14}{ }^{20}$ We contacted the authors of the remaining six studies and one author responded to supply a complete data set. ${ }^{15}$

Table 3 details the study outcomes, time points and a summary of results including adverse events. Only two of the nine trials clearly specified a primary outcome. ${ }^{19} 20$ The VAS for base of thumb pain was used by all trials, however it was used in several different formats such as the standard VAS (average of pain and activity), VAS (rest), VAS (activity), VAS (pressure) and VAS (average of pain, activity and pressure). The majority of trials final follow-up was at 6 months, the exceptions to this being the studies by Bahadir $e t a l^{2}$ and Roux $e t a l^{20}$ which followed participants until 12 and 3 months, respectively.

Table 4 describes the concomitant treatment undergone by the trial participants broken down into analgesia, splint use and other. Some trials made little mention of concomitant therapies, for example, Stahl et $a l^{15}$ made no mention of other treatments while Meenagh $e t a l^{19}$ only mentioned that a splint was used for 48 hours after the injection. The approach to analgesics was highly variable. Meenagh $e t a l^{19}$ did not mention analgesics Monfort $e t a l^{14}$ and Roux $e t a t^{20}$ allowed all analgesics while all the other trials prohibited the use of analgesics in a highly variable manner. The approach to splintage was also highly variable. Both Roux $e t a l^{20}$ and Fuchs $e t a l^{33}$ made no change to splint usage. While Jahangiri et $a l^{18}$ excluded patients who used splints and Bahadir $e t$ al $^{12}$ prohibited the use of splints. Heyworth et $a l^{17}$ and Meenagh $e t a l^{19}$ specified the use of a splint for a short period after injection therapy, but did not describe splint usage outside of this window. ${ }^{19}$ Monfort et $a l^{14}$ Stahl et $a l^{15}$ and Figen Ayhan and Ustün ${ }^{16}$ all made no mention of splint usage. Only two trials made any mention of hand therapy, Jahangiri et $a l^{14}$ instructed patients not to undergo any therapy, while Monfort $e t a l^{18}$ excluded patients who had undergone hand therapy.

\section{Adverse events}

All trials reported that no adverse events had occurred as a result of any trial interventions, thus demonstrating the general safety of injection-based therapies. However, the absence of published study protocols and published details regarding what precisely constituted an 'adverse event' surveillance does make it difficult to be specific as to what this actually means.

\section{Risk of bias within studies and across studies}

Overall, the degree of bias was fairly heterogeneous across all bias domains. Only one trial was at high risk of bias in terms of sequence generation due to the use of a sequence generated by the patient's hospital number. ${ }^{16}$ Blinding of participants was not possible in the trials by Bahadir $e t$ al and Roux $e t$ al due to the different number of injections received by both treatment groups, ${ }^{12} 20$ while the injecting clinician was not blinded in the trial by Heyworth et al. ${ }^{17}$ One study was at high risk of bias regarding incomplete outcome data due to a significantly greater loss to follow-up in the steroid injection group. ${ }^{14}$ The risk of bias summary is shown in figure 2 and the risk of bias graph is included as online supplementary file 2 . Other sources of bias included the use of a single individual performing injections in a single centre, ${ }^{12}$ industry funding ${ }^{13}{ }^{17}$ the 'random' exclusion of a large group of patients, ${ }^{18}$ underpowering by not meeting study's own stated number of patients, ${ }^{19}$ a lack of control group ${ }^{20}$ and the role of industry is providing sodium hyaluronate without charge. ${ }^{15}$

\section{Results of individual studies and synthesis of results}

The results of the individual trials are summarised in table 3. Due to limited data, meta-analysis was only performed for the comparison of corticosteroid versus hyaluronic acid for pain (ie, VAS rest and VAS activity) and function (ie, grip strength and tip pinch strength) (figures 3-6).

\section{Pain}

Corticosteroid versus hyaluronic acid

Bahadir et $a l^{12}$ demonstrated that steroid was superior to hyaluronic acid in terms of pain (VAS (activity)) in the medium term (MD -2.20, 95\% CI -3.45 to -0.95) but not at long term. Fuchs $e t a l^{13}$ showed a short term (2 and 3 weeks) superiority of steroid over hyaluronic acid in terms of pain (VAS). The studies by Heyworth $e t a l,{ }^{17}$ Monfort $e t$ $a l^{14}$ and Stahl et $a l^{15}$ showed no difference in pain in the short and medium term (figures 3 and 4).

Meta-analysis of the studies by Bahadir et al and Stahl et al showed a small reduction in pain (VAS (activity)) in medium term in those participants who received corticosteriod compared with control, however there was no difference in the short or long term (figure 3). Meta-analysis of the studies by Monfort et al and Stahl et al demonstrated no difference in pain (VAS (rest)) between corticosteroid and hyaluronic acid in the short and medium term (figure 4).

\section{Corticosteroid versus placebo}

The studies by Heyworth et $a l^{17}$ and Meenagh et $a l^{19}$ demonstrated no difference in pain (VAS) in the short and medium term, however no further analysis was possible due to the incomplete data provided. 


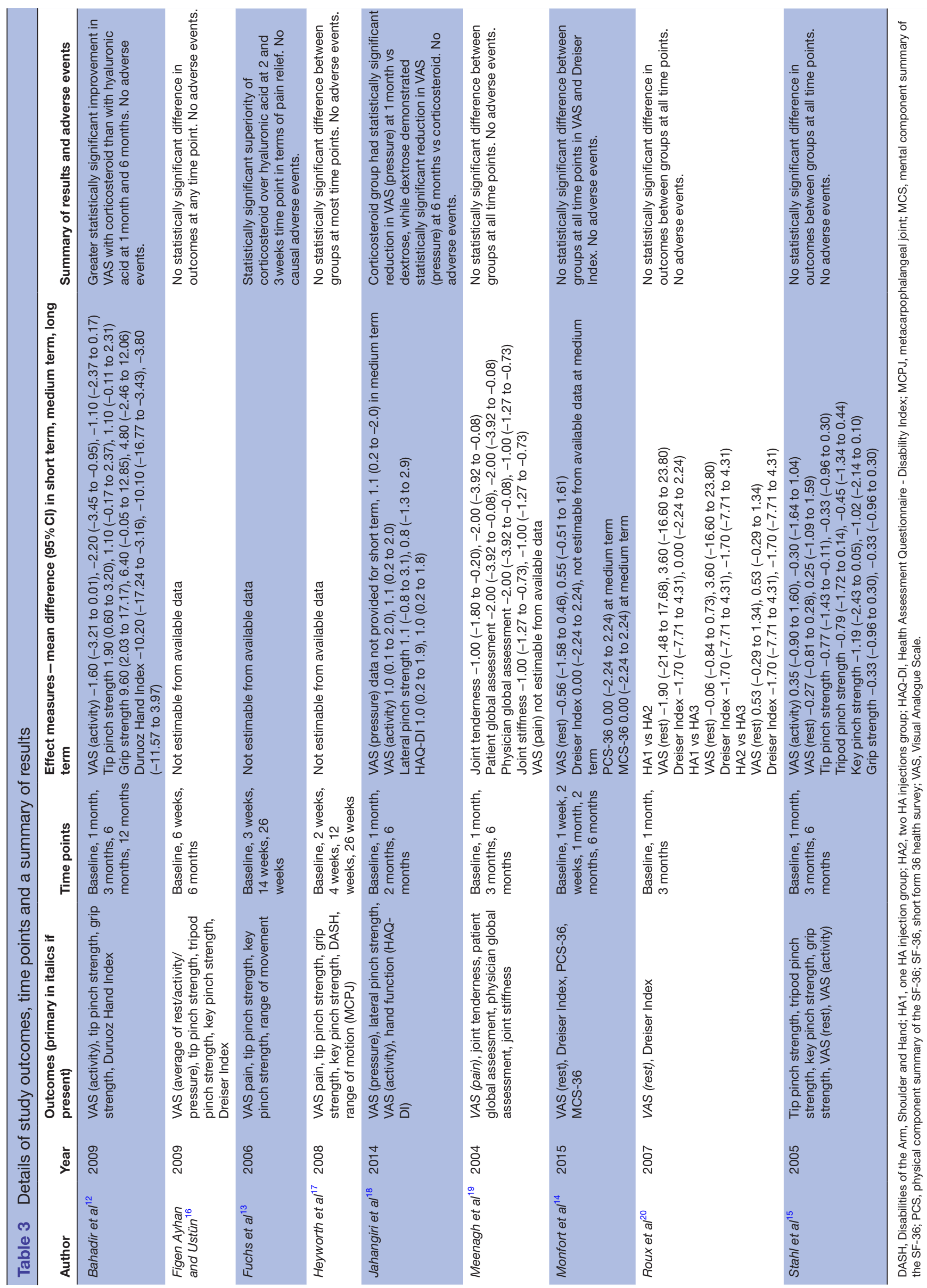




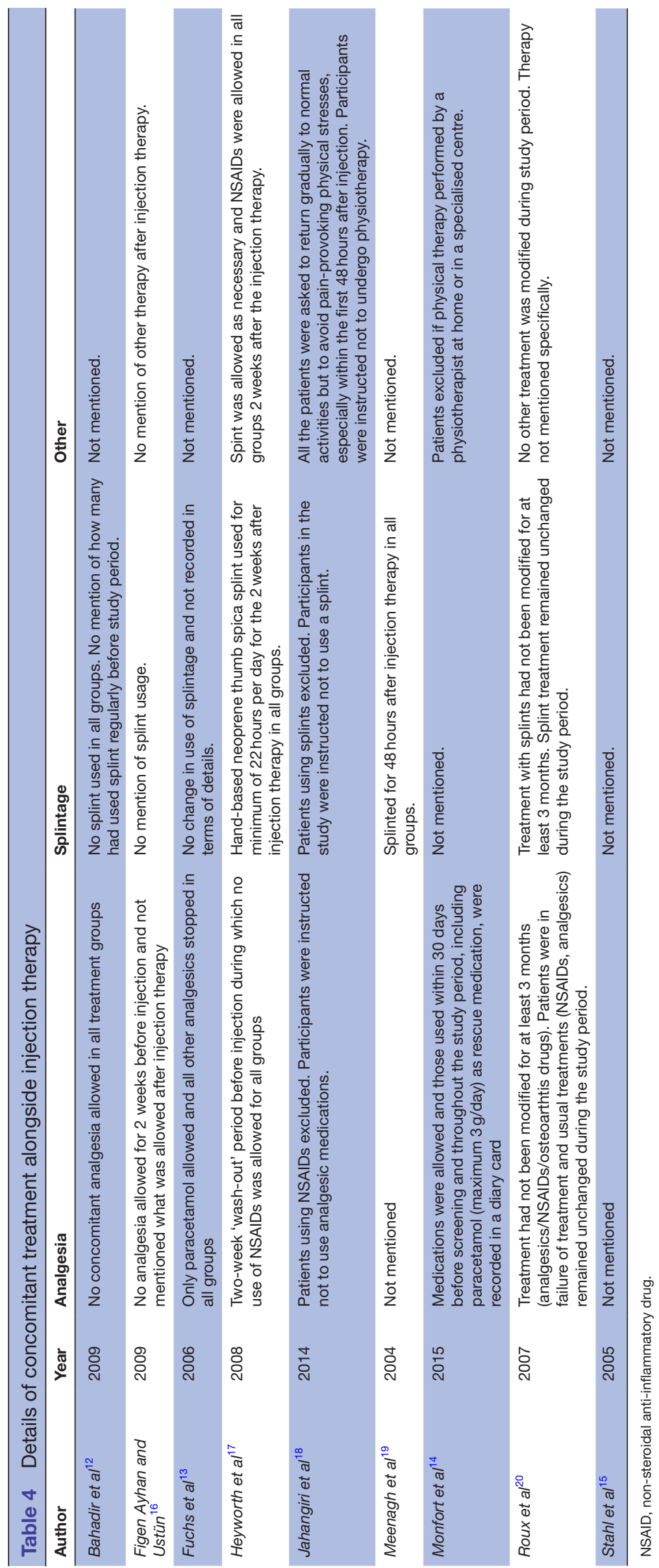

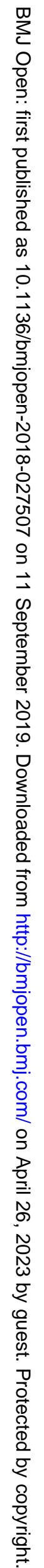




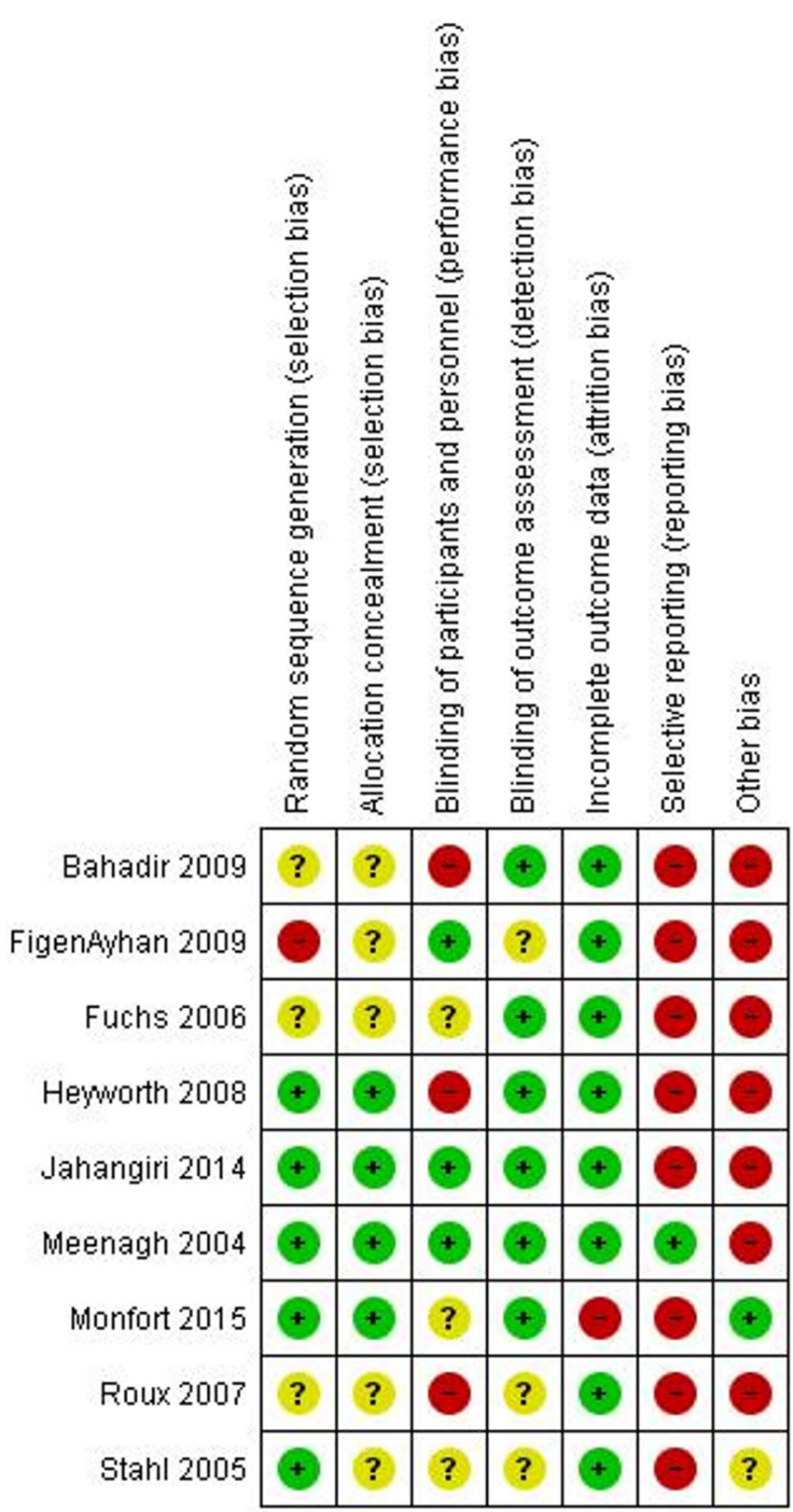

Figure 2 Risk of bias summary. Review the authors' judgements about each risk of bias item for each included study.

Hyaluronic acid versus placebo

The studies by Heyworth et $a l^{17}$ and Figen Ayhan et $a l^{16}$ demonstrated no difference in pain in the short and medium term, again no further analysis was possible due to the incomplete data provided.

\section{Corticosteroid versus dextrose}

Jahangiri et al found that the corticosteroid group had a reduction in VAS (rest) in the short term versus dextrose, however there was no difference in pain (VAS (activity)). ${ }^{18}$ Jahangiri et alalso demonstrated a reduction in pain (VAS (pressure)) in the medium term in the dextrose group compared with the corticosteroid group ${ }^{18}$ however there was no difference in pain (VAS (activity)) in the medium term.
Hyaluronic acid comparisons

Roux et al demonstrated no difference in pain (VAS (rest)) in the short term with one versus two versus three hyaluronic acid injections in the short and medium term. $^{20}$

\section{Function (tip pinch strength and grip strength) \\ Corticosteroid versus hyaluronic acid}

The studies by Heyworth et al, Monfort et al and Stahl et al showed no difference in hand function in the short term and medium term. ${ }^{141517}$ Bahadir et al demonstrated that steroid was superior to hyaluronic acid in terms of function in the short and medium term (Duruoz Hand Index), ${ }^{12}$ but no differences in tip pinch strength and grip strength in the long term. ${ }^{12}$

Meta-analysis of the results of the Stahl et al and Bahadir et al studies demonstrated no differences in tip pinch strength and grip strength in the short term and medium term (figures 5 and 6). ${ }^{1215}$

\section{Corticosteroid versus placebo}

The studies by Heyworth et al and Meenagh et al demonstrated no difference in function in the short and medium term, however no further analysis was possible due to the incomplete data provided. ${ }^{17} 19$

\section{Hyaluronic acid versus placebo}

The studies by Heyworth et al and Figen Ayhan et al demonstrated no difference in function in the short and medium term, again no further analysis was possible due to the incomplete data provided. ${ }^{1617}$

\section{Corticosteroid versus dextrose}

Jahangiri et al demonstrated no difference in function in the short term and medium term. ${ }^{18}$

Hyaluronic acid comparisons

Roux et al demonstrated no difference in function (Dreiser Index) in the short and medium term with one versus two versus three hyaluronic acid injections. ${ }^{20}$

\section{DISCUSSION}

The key finding of this systematic review is that the current evidence is equivocal regarding the use of injection therapy in base of thumb osteoarthritis, both in terms of which injection-based therapy is the most effective and in terms of whether any injection-based therapy is more effective than other non-injection-based interventions. It is of interest that there is no prospective evidence in which an injection-based therapy is compared with a non-injection-based intervention.

The existing evidence base suggests that a majority of patients who present with painful base of thumb osteoarthritis avoid surgical intervention. ${ }^{41}{ }^{22}$ However, it remains unclear as to which specific non-surgical interventions add value due to the significant methodological problems with the studies that have been carried out in this area. ${ }^{6723}$ As a result, it is likely that the non-operative 


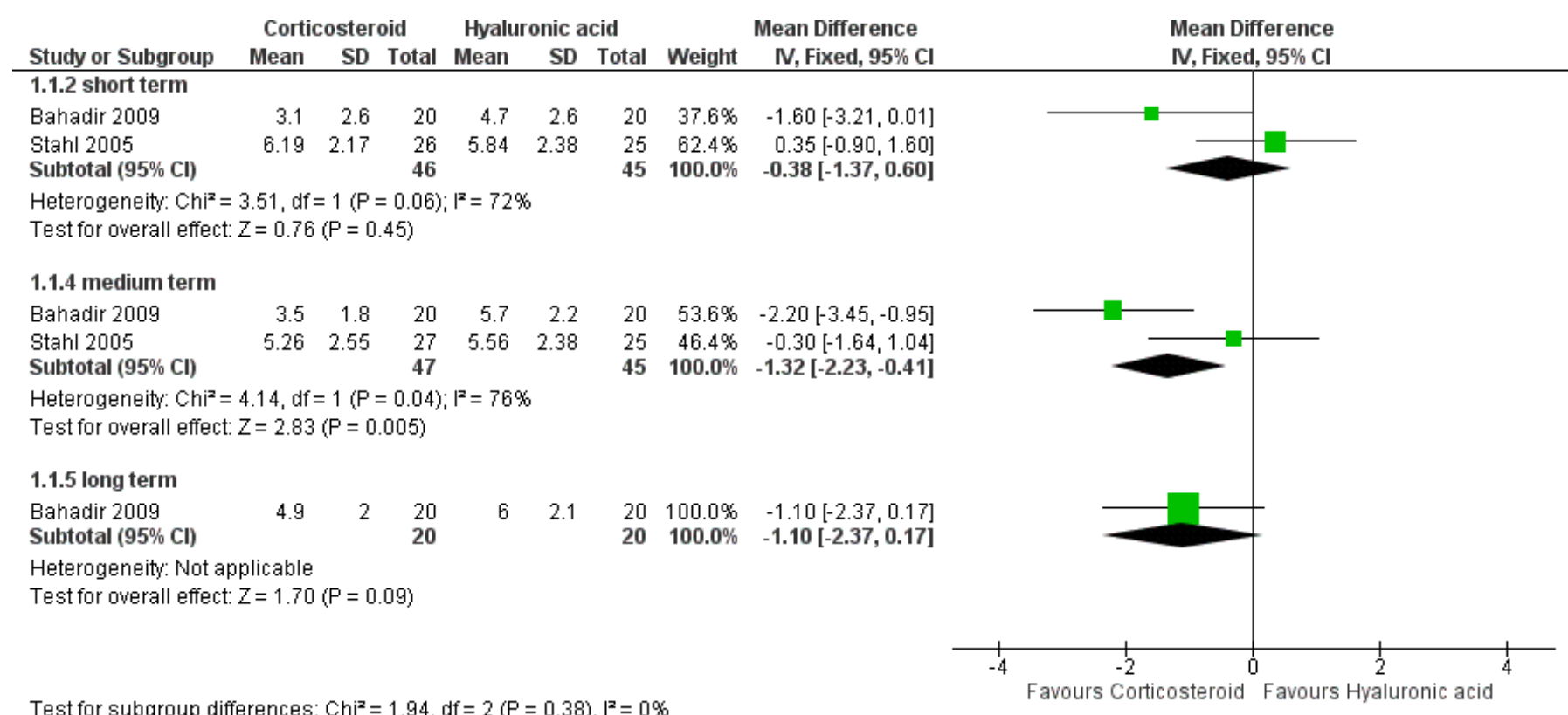

Figure 3 Forest plot of comparison: corticosteroid vs hyaluronic acid, outcome: pain-VAS (activity).

management of base of thumb osteoarthritis is highly variable, much as the surgical management appears to be. ${ }^{24}$ In the UK, corticosteroid injection is widely used, although data documenting the precise economic costs of this practice is lacking. ${ }^{25}$

A previous robust systematic review by Kroon et al has reached similar conclusions to those of our study. ${ }^{7}$ However, by obtaining additional data from the authors we were able to undertake a meta-analysis demonstrating a reduced VAS pain on activity with corticosteroid versus hyaluronic acid (MD $-1.32,95 \%$ CI -2.23 to -0.41 ) in the medium term, this being a novel finding. In this context, it is particularly difficult to justify the use of the more expensive hyaluronic acid over corticosteroid in treating base of thumb osteoarthritis. There are some other key methodological differences between our study and the review by Kroon et al. We excluded studies which had not been published in full after peer review, while these were included by Kroon et al. We have also described the approach of trials to concomitant therapies in significantly greater detail as discussed below. Broadly we feel that our findings validate and add to this previous work by Kroon $e t$ al. Overall, the justification for future research in this area remains strong, as it is imperative to determine whether such widely used interventions provide any clinically meaningful advantages over placebo.

Our review has summarised the way in which trials have handled concomitant treatments in detail and we feel this is of key importance given the way in which patients with base of thumb osteoarthritis are managed in the real world. Several studies did not even record which concomitant treatments patients had undergone before or after study interventions, while concomitant treatments were frequently managed in a rather artificial non-pragmatic manner. This can be addressed by a more pragmatic trial design as described in the recently published HIT trial protocol that has addressed the problem of concomitant treatments in hip osteoarthritis by combining

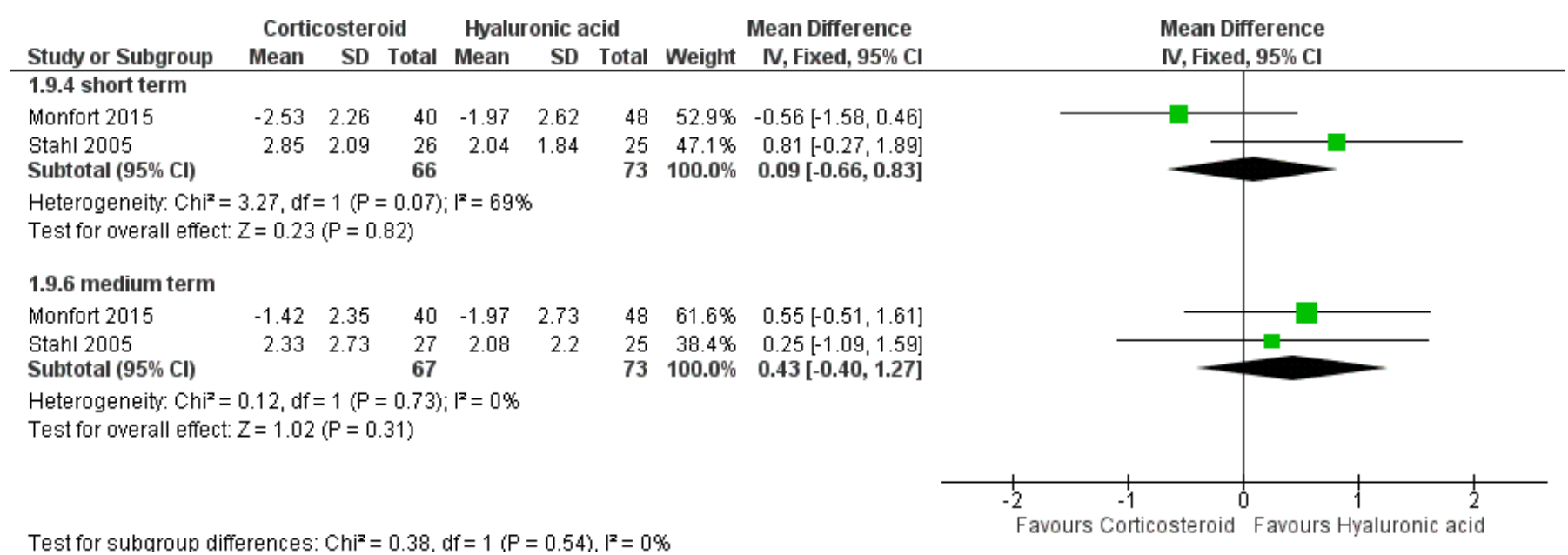

Figure 4 Forest plot of comparison: corticosteroid vs hyaluronic acid, outcome: pain -VAS (rest). 


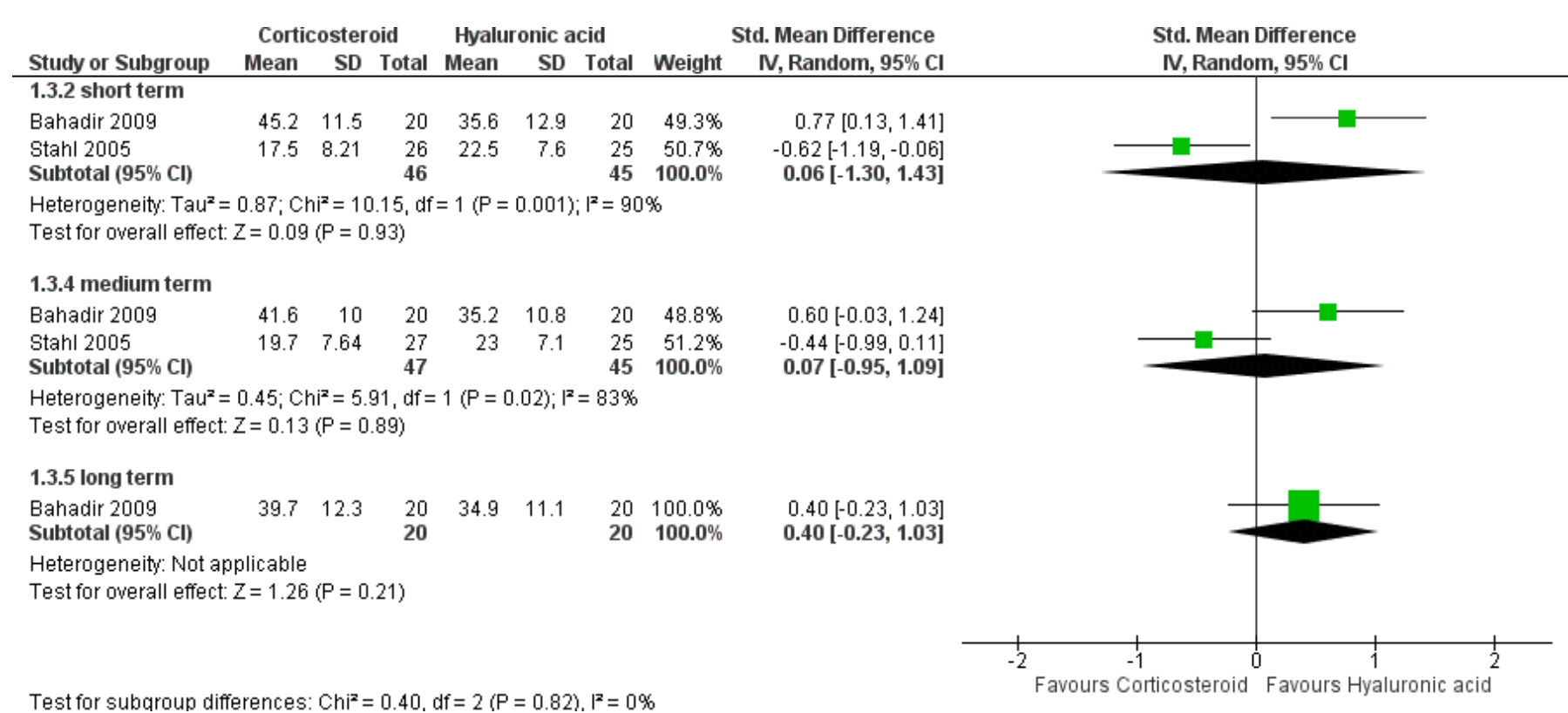

Test for subqroup differences: $\mathrm{Chi}^{2}=0.40, \mathrm{df}=2(\mathrm{P}=0.82), \mathrm{l}^{2}=0 \%$
Figure 5 Forest plot of comparison: corticosteroid vs hyaluronic acid, outcome: function - grip strength.

injection-based interventions with 'best current treatment' and ensuring that all analgesic use is recorded; in this way, the concomitant treatments become more homogenous between patients and any differences can be taken into account. ${ }^{26}$ Generally, patients in the real world are not advised to stop taking other analgesics before or after receiving an injection, ${ }^{27}$ however in several of the included studies in this review this is precisely what was done. A similar argument can be made about splint usage, as generally most patients have received some form of guidance about splint usage for symptom control before undergoing any form of injection-based intervention. Certainly, at a minimum the use of all concomitant treatments should be recorded before and after trial interventions have been administered.
Only two included studies used a specific symptom threshold for inclusion, Jahangiri et $a l^{18}$ included those with a VAS $>30 \mathrm{~mm}$ while Roux et $a l^{20}$ excluded those with a VAS $>40 \mathrm{~mm}$. The current Osteoarthritis Research Society International (OARSI) guidelines advise having a minimum cut-off for inclusion in terms of pain or function, obviously using pain or functional measures may depend on the primary outcome measure. ${ }^{28}$ This factor is another potential contributant to negative trial results as by failing to have a minimum cut-off for trial inclusion, trials are likely to have been including participants with relatively minimal levels of symptoms which makes it less likely that a clinically meaningful difference in outcomes can be achieved.

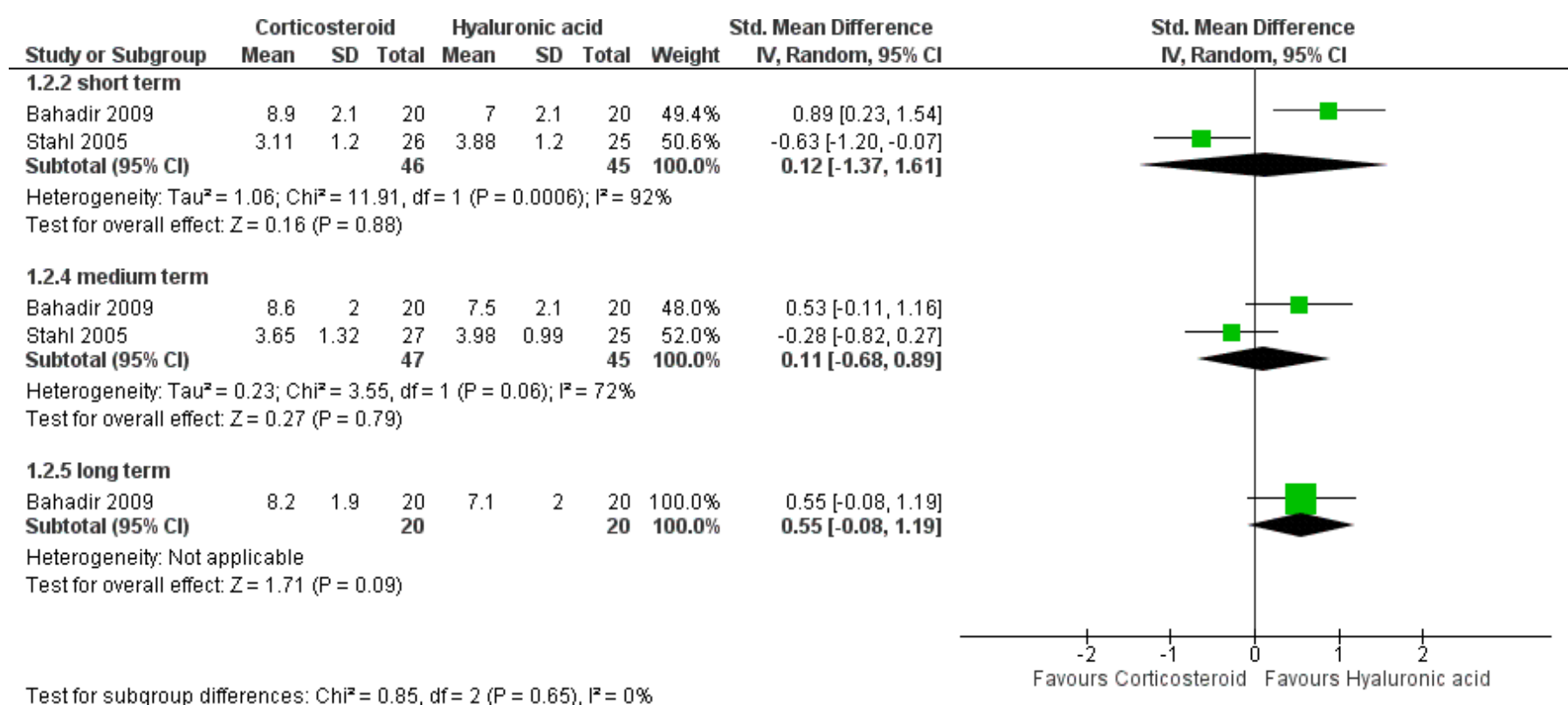

Figure 6 Forest plot of comparison: corticosteroid vs hyaluronic acid, outcome: function - tip pinch strength. 
This review has highlighted several important aspects of trial methodology which must be considered carefully in the planning and design of future research. Future trials should clearly prespecify a primary outcome measure and ideally consider current guidelines relating to clinical trials in osteoarthritis. ${ }^{28}$ Trials should involve multiple centres and be adequately powered, the current evidence base consists of virtually exclusively small single-centre studies. It is also important to ensure that the current management of base of thumb osteoarthritis is assessed in some detail, as this is also an area in which little has been published. There may be considerable variations in practice in terms of which injection therapies are used and how the injection is delivered, and in terms of the threshold for injection. This review has highlighted how variable the approach of different studies has been to dealing with the issue of concomitant or previous treatments, this also presents a challenge to researchers in the future. As discussed above, it appears best to adopt a pragmatic approach based on an assessment of what is generally deemed to be standard best practice.

\section{Limitations}

The main limitations to this systematic review and meta-analysis are the limitations intrinsic to the included studies, which are detailed above. There are several fairly consistent methodological flaws present within the trials included in this review; the studies are all small singlecentre studies which appear significantly underpowered, there is a consistent failure to clearly prespecify and state a primary outcome measure and the use of concomitant treatments has not been pragmatic. The meta-analysis has been significantly limited by a lack of adequate outcome data.

\section{CONCLUSIONS}

Current evidence is equivocal regarding the use of injection therapy in base of thumb osteoarthritis, both in terms of which injection-based therapy is the most effective and in terms of whether any injection-based therapy is more effective than other non-injection-based interventions. Given limited understanding of both the short-term and long-term effects, there is a need for large, methodologically robust multicentre RCTs investigating the commonly used injection therapies and comparison made with other therapeutic options and placebo.

\section{Twitter bendean1979}

Contributors BJFD is lead author for this review and has led the project from the start. BJFD designed the review, wrote and submitted the review protocol to PROSPERO, communicated with the research librarian who carried out the searches, carried out the screening/data extraction and data analysis and finally wrote the manuscript. MV-B and BJFD carried out the screening and data extraction. NR resolved any conflicts between BJFD and MV-B in terms of screening and data extraction. SH, AJC, MV-B and NR have been involved in the development of the study, writing the manuscript and have also reviewed the final manuscript. NT has been involved in the development of the study, carrying our the searches as well as writing and reviewing the final manuscript.
Funding This work was supported by BMA's Doris Hillier Arthritis and Rheumatism grant.

Competing interests None declared.

Patient consent for publication Not required.

Provenance and peer review Not commissioned; externally peer reviewed.

Data availability statement Data are available on reasonable request.

Open access This is an open access article distributed in accordance with the Creative Commons Attribution Non Commercial (CC BY-NC 4.0) license, which permits others to distribute, remix, adapt, build upon this work non-commercially, and license their derivative works on different terms, provided the original work is properly cited, appropriate credit is given, any changes made indicated, and the use is non-commercial. See: http://creativecommons.org/licenses/by-nc/4.0/.

\section{REFERENCES}

1. Litwic A, Edwards MH, Dennison EM, et al. Epidemiology and burden of osteoarthritis. Br Med Bull 2013;105:185-99.

2. Haugen IK, Englund M, Aliabadi P, et al. Prevalence, incidence and progression of hand osteoarthritis in the general population: the Framingham osteoarthritis study. Ann Rheum Dis 2011;70:1581-6.

3. BSSH. BSSH guidance on base of thumb osteoarthritis, 2018. Available: http://www.bssh.ac.uk/patients/conditions/24/basal_ thumb_arthritis

4. Berggren $\mathrm{M}$, Joost-Davidsson $\mathrm{A}$, Lindstrand J, et al. Reduction in the need for operation after conservative treatment of osteoarthritis of the first carpometacarpal joint: a seven year prospective study. Scand J Plast Reconstr Surg Hand Surg 2001;35:415-7.

5. Bertozzi L, Valdes K, Vanti C, et al. Investigation of the effect of conservative interventions in thumb carpometacarpal osteoarthritis: systematic review and meta-analysis. Disabil Rehabil 2015;37:2025-43.

6. Spaans AJ, van Minnen LP, Kon M, et al. Conservative treatment of thumb base osteoarthritis: a systematic review. J Hand Surg Am 2015;40:16-21.

7. Kroon FPB, Rubio R, Schoones JW, et al. Intra-Articular therapies in the treatment of hand osteoarthritis: a systematic literature review. Drugs Aging 2016;33:119-33.

8. Coombes BK, Bisset L, Vicenzino B. Efficacy and safety of corticosteroid injections and other injections for management of tendinopathy: a systematic review of randomised controlled trials. Lancet 2010;376:1751-67.

9. Coombes BK, Bisset L, Brooks P, et al. Effect of corticosteroid injection, physiotherapy, or both on clinical outcomes in patients with unilateral lateral epicondylalgia: a randomized controlled trial. JAMA 2013;309:461-9.

10. Dean BJF, Franklin SL, Murphy RJ, et al. Glucocorticoids induce specific ion-channel-mediated toxicity in human rotator cuff tendon: a mechanism underpinning the ultimately deleterious effect of steroid injection in tendinopathy? Br J Sports Med 2014;48:1620-6.

11. Higgins JPT GS. Cochrane Handbook for Systematic Reviews of Interventions Version 5.1.0 [updated March 2011]. The Cochrane Collaboration, 2011. Available: www.cochrane-handbook.org

12. Bahadır C, Onal B, Dayan VY, et al. Comparison of therapeutic effects of sodium hyaluronate and corticosteroid injections on trapeziometacarpal joint osteoarthritis. Clin Rheumatol 2009;28:529-33.

13. Fuchs $\mathrm{S}$, Mönikes $\mathrm{R}$, Wohlmeiner $\mathrm{A}$, et al. Intra-Articular hyaluronic acid compared with corticoid injections for the treatment of rhizarthrosis. Osteoarthritis Cartilage 2006;14:82-8.

14. Monfort J, Rotés-Sala D, Segalés N, et al. Comparative efficacy of intra-articular hyaluronic acid and corticoid injections in osteoarthritis of the first carpometacarpal joint: results of a 6-month single-masked randomized study. Joint Bone Spine 2015;82:116-21.

15. Stahl S, Karsh-Zafrir I, Ratzon N, et al. Comparison of intraarticular injection of depot corticosteroid and hyaluronic acid for treatment of degenerative trapeziometacarpal joints. J Clin Rheumatol 2005;11:299-302.

16. Figen Ayhan F, Ustün N. The evaluation of efficacy and tolerability of Hylan G-F 20 in bilateral thumb base osteoarthritis: 6 months followup. Clin Rheumatol 2009;28:535-41.

17. Heyworth BE, Lee JH, Kim PD, et al. Hylan versus corticosteroid versus placebo for treatment of basal joint arthritis: a prospective, randomized, double-blinded clinical trial. $J$ Hand Surg Am 2008;33:40-8.

18. Jahangiri A, Moghaddam FR, Najafi S. Hypertonic dextrose versus corticosteroid local injection for the treatment of osteoarthritis in the 
first carpometacarpal joint: a double-blind randomized clinical trial. $J$ Orthop Sci 2014;19:737-43.

19. Meenagh GK, Patton J, Kynes C, et al. A randomised controlled trial of intra-articular corticosteroid injection of the carpometacarpal joint of the thumb in osteoarthritis. Ann Rheum Dis 2004;63:1260-3.

20. Roux C, Fontas E, Breuil V, et al. Injection of intra-articular sodium hyaluronidate (Sinovial) into the carpometacarpal joint of the thumb (Cmc1) in osteoarthritis. A prospective evaluation of efficacy. Joint Bone Spine 2007;74:368-72.

21. Maarse W, Watts AC, Bain GI. Medium-Term outcome following intra-articular corticosteroid injection in first CMC joint arthritis using fluoroscopy. Hand Surg 2009;14:99-104.

22. Day CS, Gelberman R, Patel AA, et al. Basal joint osteoarthritis of the thumb: a prospective trial of steroid injection and splinting. $J$ Hand Surg Am 2004;29:247-51.

23. Fowler A, Swindells MG, Burke FD. Intra-Articular corticosteroid injections to manage trapeziometacarpal osteoarthritis-a systematic review. Hand 2015;10:583-92.
24. Brunton LM, Wilgis EFS. A survey to determine current practice patterns in the surgical treatment of advanced thumb carpometacarpal osteoarthrosis. Hand 2010;5:415-22.

25. Anakwe RE, Middleton SD. Osteoarthritis at the base of the thumb. BMJ 2011;343.

26. Paskins $Z$, Hughes $G$, Myers $\mathrm{H}$, et al. A randomised controlled trial of the clinical and cost-effectiveness of ultrasound-guided intra-articular corticosteroid and local anaesthetic injections: the hip injection trial (HIT) protocol. BMC Musculoskelet Disord 2018;19:218.

27. VersusArthritis. Guidance regarding steroid injections, 2019. Available: https://www.versusarthritis.org/about-arthritis/treatments/ drugs/steroid-injections/

28. Kloppenburg M, Maheu E, Kraus VB, et al. OARSI clinical trials recommendations: design and conduct of clinical trials for hand osteoarthritis. Osteoarthritis Cartilage 2015;23:772-86.

29. Moher D, Liberati A, Tetzlaff J, et al. Preferred reporting items for systematic reviews and meta-analyses: the PRISMA statement. PLoS Med 2009;6:e1000097. 